\title{
Comparison of Antiplatelet and Antithrombotic Therapy for Secondary Prevention of Ischemic Stroke in Patients With Peripheral Artery Disease
}

- Population-Based Follow-up Study in Taiwan -

\author{
Wen-Hsien Lee, MD; Chun-Yuan Chu, MD; Po-Chao Hsu, MD; \\ Ho-Ming Su, MD; Tsung-Hsien Lin, MD, PhD; Wen-Chol Voon, MD; \\ Wen-Ter Lai, MD; Sheng-Hsiung Sheu, MD
}

\begin{abstract}
Background: A limited number of studies have assessed the benefit and risk among the different antiplatelet and antithrombotic therapies in patient with stroke and peripheral artery disease (PAD). We compared the efficacy and safety of clopidogrel, cilostazol, warfarin, and aspirin.

Methods and Results: A retrospective cohort study analyzing the Taiwan National Health Insurance Research Dataset identified patients with stroke and PAD from 2002 to 2008. Patients were stratified according to their use of aspirin, clopidogrel, cilostazol, warfarin or combination therapy. A total of 1,686 patients were enrolled: aspirin $(n=862)$, clopidogrel $(n=92)$, warfarin $(n=136)$, cilostazol only $(n=515)$, and cilostazol-based combination therapy $(n=81)$. Compared with aspirin, cilostazol could reduce the risk of ischemic stroke [hazard ratio $(\mathrm{HR}) 0.79,95 \%$ confidence interval $(\mathrm{Cl}) 0.63-0.98, \mathrm{P}=0.0349)$ and no increase in hemorrhagic events $(\mathrm{HR} 0.98,95 \% \mathrm{Cl} 0.74-1.32$, $\mathrm{P}=0.9122)$. Clopidogrel decreased the risk of ischemic stroke (HR $0.47,95 \% \mathrm{Cl} 0.29-0.78, \mathrm{P}=0.0033$ ) and hemorrhagic events (HR $0.64,95 \% \mathrm{Cl} 0.31-0.96, \mathrm{P}=0.034)$ more than aspirin. There was no statistical difference regarding the risk of stroke and hemorrhagic events among warfarin, cilostazol-based combination therapy and aspirin.
\end{abstract}

Conclusions: Cilostazol and clopidogrel were more effective in preventing recurrent ischemic stroke without increased hemorrhagic events than aspirin in patients with PAD. (Circ $J$ 2013; 77: 1046-1052)

Key Words: Aspirin; Cilostazol; Clopidogrel; Peripheral artery disease; Stroke

$\mathbf{C}$ erebrovascular disease (CVD) can cause several physical and mental disabilities and results in a great economic burden on the Taiwanese healthcare system. ${ }^{1-3}$ In previous epidemiological studies, the major type of CVD has been cerebral infarction and $66 \%$ of the causes of acute cerebral infarction were large artery atherosclerosis, lacunae and cardiogenic embolism.., 5 Current treatments, including antiplatelet agents and anticoagulants, for prevention of stroke should be based on the underlying pathophysiology.

In the REACH (REduction of Atherothrombosis for Continued Health) registry, patients with more than 1 vascular bed affected by atherosclerotic disease, such as CVD plus CAD (coronary artery disease) and/or PAD (peripheral artery disease), had higher rates of cardiovascular and cerebrovascular events than patients with CVD alone. ${ }^{6,7}$ In patient with ischemic stroke, a lower ankle-brachial index (ABI) was found to be associated with poor CVD outcome, more severe carotid artery stenosis, and a higher rate of cardiovascular events and death. ${ }^{8-10}$ Therefore, it is mandatory to prevent recurrent cerebrovascular events in patients with coexisting CVD and PAD.

Antiplatelet agents play an important role in preventing atherothrombotic events. Aspirin and clopidogrel are effective for protection of atherothrombotic disease in high-risk populations. ${ }^{11,12}$ Cilostazol is an antiplatelet agent that inhibits platelet aggregation via inhibition of cyclic AMP phosphodiesterase III and increase in cyclic AMP concentrations. ${ }^{13}$ It is indicated in PAD patients with intermittent claudication. ${ }^{14}$ In the recent Cilostazol Stroke Prevention Study (CSPS) and CSPS

Received August 30, 2012; revised manuscript received October 17, 2012; accepted November 27, 2012; released online December 26, 2012 Time for primary review: 19 days

Division of Cardiology, Department of Internal Medicine, Kaohsiung Medical University Hospital, Kaohsiung (W.-H.L., C.-Y.C., P.-C.H., H.-M.S., T.-H.L., W.-C.V., W.-T.L., S.-H.S.); Department of Internal Medicine, Kaohsiung Municipal Hsiao-Kang Hospital, Kaohsiung (W.-H.L., H.-M.S.); and Department of Internal Medicine, Faculty of Medicine, College of Medicine, Kaohsiung Medical University, Kaohsiung (H.-M.S., T.-H.L., W.-C.V., W.-T.L., S.-H.S.), Taiwan

Mailing address: Tsung-Hsien Lin, MD, PhD, Division of Cardiology, Department of Internal Medicine; Kaohsiung Medical University Hospital, 100 Tzyou 1st Road, Kaohsiung, 80708, Taiwan, ROC. E-mail: 1th@kmu.edu.tw

ISSN-1346-9843 doi:10.1253/circj.CJ-12-1122

All rights are reserved to the Japanese Circulation Society. For permissions, please e-mail: cj@j-circ.or.jp 


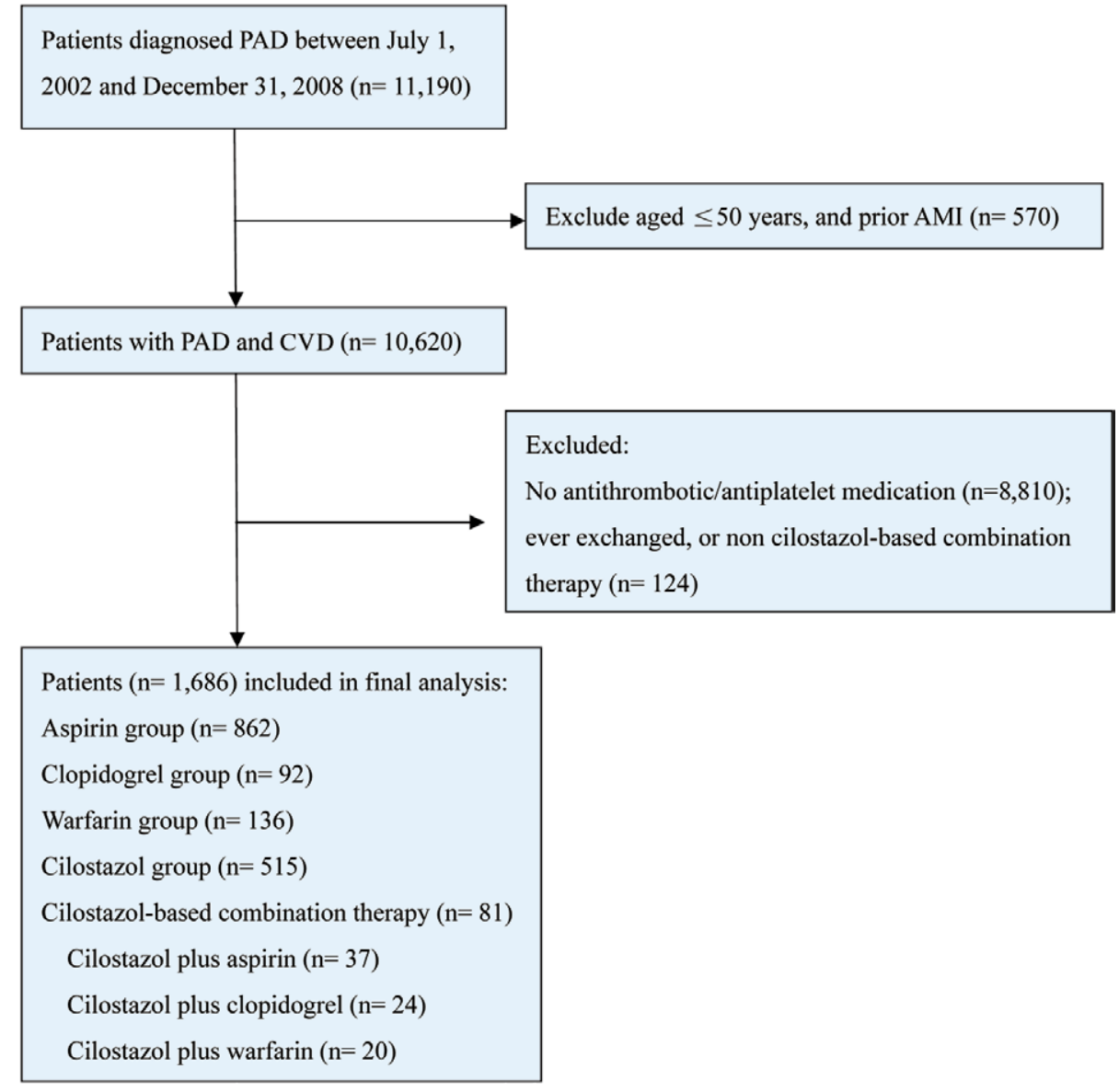

Figure. Flow chart of study. AMI, acute myocardial infarction; CVD, cerebrovascular disease; PAD, peripheral artery disease.

2 trials, it was more effective and safe than aspirin and placebo for the secondary prevention of non-cardioembolic ischemic stroke in Asian populations. ${ }^{15,16}$ Although anticoagulant therapy can reduce major cardiovascular events in patients with $\mathrm{CAD}$, its benefit for intermittent claudication and risk reduction of vascular ischemic events in the PAD population is not established. ${ }^{14,17}$ Therefore, we investigated the efficacy and safety of aspirin, clopidogrel, cilostazol, warfarin, and cilostazol-based combination therapy for secondary prevention of ischemic stroke in patients with PAD.

\section{Methods}

\section{Data Source}

Data from the National Health Insurance Research Dataset (NHIRD), published by the National Health Research Institute (NHI) in Taiwan, which provided a database of 1,000,000 random subjects, were analyzed. The NHI program has been operating in Taiwan since 1995, offering a comprehensive, unified, and universal health insurance program to all citizens who have established a registered domicile for at least 4 months in Taiwan. The coverage includes outpatient service, inpatient care, Chinese medicine, dental care, childbirth, physical therapy, preventive health care, home care, and rehabilitation for chronic mental illness. In $2000,96.16 \%$ of the whole popula- tion were covered and by the end of 2004 it had increased to $99 \%$. The NHI medical claims database includes ambulatory care, hospital inpatient care, dental services, and prescription drugs. Therefore, the NHIRD is one of the largest and most complete nationwide population-based datasets in Taiwan and there were no statistically significant differences in age, sex, and average insured payroll-related amount between the sample group and all enrollees.

\section{Study Sample}

This study population consisted of all patients with more than 2 outpatient or inpatients claims with a diagnosis of PAD (ICD-9-CM: 250.7, 443, 443.81, 443.9, 444.2, 785.4) and prior stroke (ICD-9-CM: 430-438) between July 1, 2002, and December 31, 2008. The data of the first claim with a PAD diagnosis was considered the index date. Patients younger than 50 years and with any diagnosis of acute myocardial infarction (AMI; ICD-9-CM: 410) before the index date were excluded. Claims for antithrombotic or antiplatelet treatment were identified in the 120 days after each patient's index date. ${ }^{18}$ Antithrombotic or antiplatelet medication was classified as aspirin, clopidogrel, warfarin, cilostazol, or cilostazol-based combination therapy. The aspirin, clopidogrel, warfarin, and cilostazol groups were defined as use of a single medication for treatment during the follow-up. The cilostazol-based combination 


\begin{tabular}{|lccccccr|}
\hline Table 1. Characteristics of the Study Population & & & & \\
Variable, $\mathbf{n}(\%)$ & $\begin{array}{c}\text { Total cases } \\
(\mathbf{n = 1 , 6 8 6 )}\end{array}$ & $\begin{array}{c}\text { Aspirin } \\
\mathbf{( n = 8 6 2 )}\end{array}$ & $\begin{array}{c}\text { Clopidogrel } \\
(\mathbf{n}=\mathbf{9 2})\end{array}$ & $\begin{array}{c}\text { Warfarin } \\
(\mathbf{n = 1 3 6 )}\end{array}$ & $\begin{array}{c}\text { Cilostazol } \\
(\mathbf{n}=515)\end{array}$ & $\begin{array}{c}\text { Combination } \\
(\mathbf{n}=\mathbf{8 1})\end{array}$ & P value \\
Sex & & & & & & & 0.2777 \\
$\quad$ Female & $855(50.7)$ & $454(52.7)$ & $42(45.7)$ & $74(54.4)$ & $245(47.6)$ & $40(49.4)$ & \\
$\quad$ Male & $831(49.3)$ & $408(47.3)$ & $50(54.3)$ & $62(45.6)$ & $270(52.4)$ & $41(50.6)$ & \\
Age & & $68.68 \pm 9.74$ & $70.79 \pm 9.01$ & $68.34 \pm 9.73$ & $69.52 \pm 10.03$ & $70.69 \pm 10.12$ & 0.4014 \\
CAD & $445(26.4)$ & $247(28.7)$ & $40(43.5)$ & $36(26.5)$ & $102(19.8)$ & $20(24.7)$ & $<0.0001$ \\
CHF & $104(6.2)$ & $39(4.5)$ & $13(14.1)$ & $19(14.0)$ & $25(4.9)$ & $8(9.9)$ & $<0.0001$ \\
Hypertension & $1,027(60.8)$ & $558(64.7)$ & $66(71.7)$ & $68(50.0)$ & $294(57.1)$ & $41(50.6)$ & 0.0001 \\
AF & $45(2.7)$ & $9(1.0)$ & $4(4.3)$ & $20(14.7)$ & $8(1.6)$ & $4(4.9)$ & $<0.0001$ \\
Hyperlipidemia & $406(24.1)$ & $220(25.5)$ & $34(37.0)$ & $21(15.4)$ & $113(21.9)$ & $18(22.2)$ & 0.0026 \\
DM & $826(49.0)$ & $414(48.0)$ & $55(59.8)$ & $41(30.1)$ & $272(52.8)$ & $44(54.3)$ & $<0.0001$ \\
COPD & $112(6.6)$ & $65(7.5)$ & $4(4.3)$ & $5(3.7)$ & $33(6.4)$ & $5(6.2)$ & 0.4189 \\
CKD & $233(13.8)$ & $77(8.9)$ & $25(27.2)$ & $27(19.9)$ & $86(16.7)$ & $18(22.2)$ & $<0.0001$ \\
Prior GI event & $218(12.9)$ & $87(10.1)$ & $29(31.5)$ & $16(11.8)$ & $68(13.2)$ & $18(22.2)$ & $<0.0001$ \\
PPI use & $66(3.9)$ & $24(2.8)$ & $10(10.9)$ & $5(3.7)$ & $18(3.5)$ & $9(11.1)$ & $<0.0001$ \\
\hline
\end{tabular}

$\mathrm{AF}$, atrial fibrillation; CAD, coronary artery disease; CHF, congestive heart failure; DM, diabetes mellitus; COPD, chronic obstructive pulmonary disease; CKD, chronic kidney disease; GI, gastrointestinal; PPI, proton-pump inhibitor.

therapy group was defined as cilostazol combined with aspirin, clopidogrel, or warfarin in 1 prescription. Patients' sex, age, and treatment information were obtained, and comorbidities were extracted from all claims within the 180 days before the index data.

\section{Study Outcomes}

The outcome factors included ischemic stroke (ICD-9-CM: 433-435), hemorrhagic stroke (ICD-9-CM: 430-432), other stroke (436-438), all stroke (including ischemic, hemorrhagic, and other stroke), and hospitalization due to AMI (ICD-9-CM: 410), heart failure (ICD-9-CM: 428) and gastrointestinal (GI) events (including GI hemorrhage, gastric ulcer, duodenal ulcer, and peptic ulcer; ICD-9-CM: 578, 531, 532, 533).

\section{Statistical Analysis}

The chi-square test was used to compare the distribution of sociodemographic characteristics between different treatments. Time-to-event analysis involved estimating the probability that an event would occur at different points in time. The followup time for survival started at the date of diagnosis and ended at the date of different categories developed or last observation up to December 31, 2008.

Two sets of hazard ratios (HRs) were computed for analysis factors by Cox regression analyses. The univariate HRs were estimated from separate Cox regressions with 1 analysis factor at a time. The multivariable-adjusted HRs were computed from Cox regression with additional variables of sex (men and women), age, CAD (ICD-9-CM: 411-414), congestive heart failure (CHF; ICD-9-CM: 428), hypertension (ICD-9-CM: 401-405), atrial fibrillation (AF; ICD-9-CM: 427.31), hyperlipidemia (ICD-9-CM: 272.0-272.4), diabetes mellitus (DM; ICD-9-CM: 250), chronic obstructive pulmonary disease (COPD; ICD-9-CM: 491, 492, 496), chronic kidney disease (CKD; ICD-9-CM:250.4, 274.1, 283.11, 403, 404, 440.1, 442.1, 447.3, 572.4, 580-588, 642.1, 646.2) and PPI (proton-pump inhibitors). Significance was set at $\mathrm{P}<0.05$. All the data processing and statistical analysis were performed with SAS 9.2 software (Cary, NC, USA).

\section{Results}

We identified 11,190 patients with PAD from January 2002 to December 2008. After excluding those patients who did not fit the inclusion criteria, a total of 1,686 patients ( $49.3 \%$ male) were included in the final analysis (Figure). Table 1 presents the baseline characteristics of the aspirin, clopidogrel, cilostazol, warfarin, and cilostazol-based combination therapy groups. There was a statistically significant difference regarding $\mathrm{CAD}, \mathrm{CHF}$, hypertension, $\mathrm{AF}$, hyperlipidemia, diabetes, $\mathrm{CKD}$, prior GI events and PPI use among the groups. There were more comorbidities, including $\mathrm{CAD}, \mathrm{CHF}$, hypertension, hyperlipidemia, DM, CKD and prior GI events, in the clopidogrel group.

Table 2 presents crude adjusted HRs and $95 \%$ confidence intervals (CIs) of hemorrhagic, ischemic, other stroke and all strokes among the 4 groups by conditional logistic regression. Compared with the aspirin group, the risks of other stroke (crude HR 0.70, 95\% CI 0.57-0.87), and all stroke (crude HR $0.77,95 \%$ CI $0.65-0.92$ ) were significant lower in the cilostazol group. After adjustment for possible confounders (age, sex, CAD, CHF, hypertension, AF, hyperlipidemia, DM, COPD, CKD, prior GI events, PPI use, the risks of ischemic stroke (adjusted HR 0.79, 95\% CI 0.63-0.98), other stroke (adjusted HR 0.66, 95\% CI 0.53-0.82), and all stroke (adjusted HR $0.74,95 \%$ CI 0.62-0.88) were significantly lower in the cilostazol group than in the aspirin group. In contrast, the risks of ischemic stroke (crude HR 1.79, 95\% CI 1.312.45 ), other stroke (crude HR 1.58, 95\% CI 1.15-2.16) and all stroke (crude HR 1.66, 95\% CI 1.27-2.18) were significantly higher in the clopidogrel group than in the aspirin group. After adjustment for possible confounders, the risks of ischemic stroke (adjusted HR 1.47, 95\% CI 1.07-2.04), and all stroke (adjusted HR 1.34, 95\% CI 1.01-1.78) were still significantly higher in the clopidogrel group than in the aspirin group. There were no significant differences among the warfarin, cilostazol-based combination and aspirin groups for hemorrhagic, ischemic stroke, other stroke and all strokes.

With regard to safety, we analyzed AMI, GI events, and all hemorrhagic events (including hemorrhagic stroke and GI events) among the 4 groups (Table 3 ). There were no signifi- 


\begin{tabular}{|c|c|c|c|c|c|}
\hline & $\begin{array}{l}\text { Aspirin } \\
(n=862)\end{array}$ & $\begin{array}{c}\text { Clopidogrel } \\
(n=92)\end{array}$ & $\begin{array}{c}\text { Warfarin } \\
(n=136)\end{array}$ & $\begin{array}{c}\text { Cilostazol } \\
(n=515)\end{array}$ & $\begin{array}{c}\text { Combination* } \\
\qquad(n=81)\end{array}$ \\
\hline \multicolumn{6}{|l|}{ Hemorrhagic stroke } \\
\hline n (\%) & $36(4.2)$ & $6(6.5)$ & $6(4.4)$ & $13(2.5)$ & $5(6.2)$ \\
\hline Crude HR $(95 \% \mathrm{Cl})$ & 1.00 & $1.7(0.72-4.05)$ & $1.00(0.42-2.38)$ & $0.85(0.45-1.61)$ & $2.22(0.86-5.69)$ \\
\hline Adjusted HR (95\% Cl) & 1.00 & $1.69(0.69-4.16)$ & $1.07(0.43-2.66)$ & $0.84(0.44-1.62)$ & $2.34(0.90-6.09)$ \\
\hline$P$ value & - & 0.2541 & 0.8917 & 0.6086 & 0.08 \\
\hline \multicolumn{6}{|l|}{ Ischemic stroke } \\
\hline $\mathrm{n}(\%)$ & $282(32.7)$ & $46(50.0)$ & $47(34.6)$ & 118 (22.9) & $26(32.1)$ \\
\hline Crude HR $(95 \% \mathrm{Cl})$ & 1.00 & $1.79(1.31-2.45)$ & $1.04(0.76-1.42)$ & $0.81(0.66-1.01)$ & $1.24(0.83-1.85)$ \\
\hline Adjusted HR (95\% Cl) & 1.00 & $1.47(1.07-2.04)$ & $1.13(0.82-1.56)$ & $0.79(0.63-0.98)$ & $1.17(0.78-1.77)$ \\
\hline$P$ value & - & 0.0193 & 0.4532 & 0.0349 & 0.4422 \\
\hline \multicolumn{6}{|l|}{ Other stroke } \\
\hline $\mathrm{n}(\%)$ & $310(36.0)$ & $45(48.9)$ & $52(38.2)$ & $119(23.1)$ & $23(28.4)$ \\
\hline Crude HR $(95 \% \mathrm{Cl})$ & 1.00 & $1.58(1.15-2.16)$ & $1.05(0.78-1.41)$ & $0.70(0.57-0.87)$ & $0.95(0.62-1.44)$ \\
\hline Adjusted HR $(95 \% \mathrm{Cl})$ & 1.00 & $1.38(1.01-1.92)$ & $1.10(0.81-1.50)$ & $0.66(0.53-0.82)$ & $0.88(0.57-1.35)$ \\
\hline$P$ value & - & 0.0513 & 0.5288 & 0.0002 & 0.5455 \\
\hline \multicolumn{6}{|l|}{ All stroke } \\
\hline $\mathrm{n}(\%)$ & $419(48.6)$ & $60(65.2)$ & $63(46.3)$ & $182(35.3)$ & $34(42.0)$ \\
\hline Crude HR $(95 \% \mathrm{Cl})$ & 1.00 & $1.66(1.27-2.18)$ & $0.93(0.71-1.21)$ & $0.77(0.65-0.92)$ & $1.02(0.72-1.45)$ \\
\hline Adjusted HR (95\% Cl) & 1.00 & $1.34(1.01-1.78)$ & $1.01(0.76-1.33)$ & $0.74(0.62-0.88)$ & $0.96(0.67-1.37)$ \\
\hline$P$ value & - & 0.0413 & 0.9722 & 0.0008 & 0.8081 \\
\hline
\end{tabular}

Some patients had 2 types of stroke and therefore the numbers for all stroke are less than the numbers of ischemic plus hemorrhagic plus other stroke.

${ }^{*}$ Cilostazol-based combination therapy. $\mathrm{Cl}$, confidence interval; HR, hazard ratio.

\begin{tabular}{|c|c|c|c|c|c|}
\hline & $\begin{array}{l}\text { Aspirin } \\
(n=862)\end{array}$ & $\begin{array}{c}\text { Clopidogrel } \\
(n=92)\end{array}$ & $\begin{array}{c}\text { Warfarin } \\
(n=136)\end{array}$ & $\begin{array}{c}\text { Cilostazol } \\
(n=515)\end{array}$ & $\begin{array}{c}\text { Combination* } \\
\qquad(n=81)\end{array}$ \\
\hline \multicolumn{6}{|l|}{ AMI } \\
\hline $\mathrm{n}(\%)$ & $40(4.6)$ & $5(5.4)$ & $5(3.7)$ & $15(2.9)$ & $3(3.7)$ \\
\hline Crude HR $(95 \% \mathrm{Cl})$ & 1.00 & $1.38(0.54-3.50)$ & $0.73(0.29-1.85)$ & $1.01(0.55-1.85)$ & $1.36(0.42-4.44)$ \\
\hline Adjusted HR $(95 \% \mathrm{Cl})$ & 1.00 & $1.04(0.39-2.79)$ & $0.79(0.31-2.06)$ & $0.97(0.53-1.8)$ & $1.27(0.38-4.17)$ \\
\hline$P$ value & - & 0.9407 & 0.6361 & 0.9341 & 0.6987 \\
\hline \multicolumn{6}{|l|}{ GI events } \\
\hline $\mathrm{n}(\%)$ & $126(14.6)$ & $29(31.5)$ & $29(21.3)$ & $60(11.7)$ & $12(14.8)$ \\
\hline Crude HR $(95 \% \mathrm{Cl})$ & 1.00 & $2.7(1.8-4.05)$ & $1.42(0.95-2.12)$ & $1.15(0.84-1.56)$ & $1.50(0.93-2.72)$ \\
\hline Adjusted HR $(95 \% \mathrm{Cl})$ & 1.00 & $1.78(1.16-2.75)$ & $1.32(0.86-2.01)$ & $1.03(0.75-1.41)$ & $1.20(0.66-2.19)$ \\
\hline$P$ value & - & 0.0085 & 0.2041 & 0.8587 & 0.5548 \\
\hline \multicolumn{6}{|l|}{ All hemorrhagic events } \\
\hline $\mathrm{n}(\%)$ & $152(17.6)$ & $33(35.9)$ & $32(23.5)$ & $70(13.6)$ & $16(19.8)$ \\
\hline Crude HR $(95 \% \mathrm{Cl})$ & 1.00 & $2.58(1.77-3.76)$ & $1.31(0.89-1.92)$ & $1.08(0.81-1.43)$ & $1.68(1.01-2.82)$ \\
\hline Adjusted HR (95\% Cl) & 1.00 & $1.77(1.18-2.65)$ & $1.24(0.83-1.85)$ & $0.98(0.74-1.32)$ & $1.41(0.83-2.38)$ \\
\hline$P$ value & - & 0.0054 & 0.2919 & 0.9122 & 0.2032 \\
\hline
\end{tabular}

${ }^{*}$ Cilostazol-based combination therapy. AMI, acute myocardial infarction. Other abbreviations as in Tables 1,2.

cant differences for AMI (adjusted HR 0.97, 95\% CI 0.531.8), GI events (adjusted HR 1.03, 95\% CI 0.75-1.41) and all hemorrhagic events (adjusted HR 0.98, 95\% CI 0.74-1.32) between the cilostazol and aspirin groups. There was also no difference for AMI (adjusted HR 0.79, 95\% CI 0.31-2.06), and GI events (adjusted HR 1.32, 95\% CI 0.86-2.01) and all hemorrhagic events (adjusted HR 1.24, 95\% CI 0.83-1.85) between the warfarin and aspirin groups. The risks of GI events (adjusted HR 1.78, 95\% CI 1.16-2.75) and all hemorrhagic events (adjusted HR 1.77, 95\% CI 1.18-2.65) were significantly higher in the clopidogrel group than in the aspirin group. There was no statistical significance with regard to AMI (adjusted HR 1.27, 95\% CI 0.38-4.17), GI events (adjusted HR 1.20, 95\% CI 0.66-2.19) and all hemorrhagic events (adjusted HR 1.41, 95\% CI 0.83-2.38) between the cilostazolbased combination therapy and aspirin groups.

Because of more comorbidities in the clopidogrel group, we used the modified Charlson Comorbidity Index score matching analysis of the aspirin and clopidogrel groups (Table 4). The risks of ischemic stroke (adjusted HR 0.47, 95\% CI 0.29- 


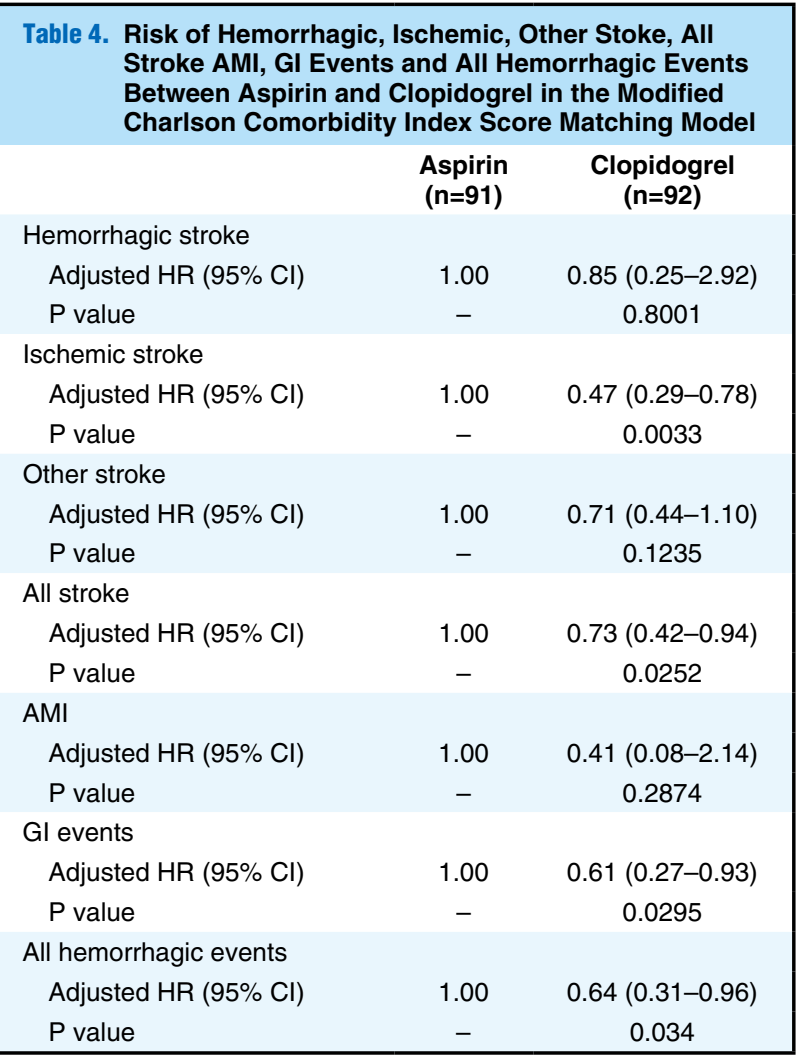

Abbreviations as in Tables 1-3.

0.78), all stroke (adjusted HR 0.73, 95\% CI 0.42-0.94), GI events (adjusted HR 0.61, 95\% CI 0.27-0.93) and all hemorrhagic events (adjusted HR 0.64, 95\% CI 0.31-0.96) were significantly lower in the clopidogrel group than in the aspirin group in this model.

\section{Discussion}

There are 3 major findings from this study. First, cilostazol significantly reduced the risk of ischemic stroke with similar safety to aspirin. Second, clopidogrel also significantly reduced the risk of ischemic stroke and was safer than aspirin. Third, there was no statistical significance for any stroke, AMI, GI and hemorrhagic events for warfarin or cilostazol-based combination therapy compared with aspirin.

Cilostazol has been shown in some clinical trials to be effective and safe for the secondary prevention of ischemic stroke, especially in Asian populations. In the CSPS (cilostazol vs. aspirin for secondary ischemic stroke prevention) trial in Japan, cilostazol was significantly associated with a $41.7 \%$ relative risk reduction in recurrent cerebral infarction without an increase in adverse drug reactions. ${ }^{16}$ However, cilostazol was only as effective as aspirin in the prevention of secondary ischemic stroke for Chinese patients in the CASISP (Cilostazol vs. Aspirin for Secondary Ischemic Stroke Prevention) and Korean patients in the CAIST (Cilostazol in Acute Ischemic Stroke Treatment) trials. It significantly reduced the rate of recurrent stroke and had lower hemorrhagic events compared with aspirin in the CSPS 2 trial in Japan. ${ }^{15,19,20}$ Our results similarly showed that cilostazol significantly reduced $(21 \%)$ the risk of ischemic stroke, with similar safety to aspirin for Taiwanese patients with PAD and CVD. We consider that the reduced risk of recurrent stroke with cilostazol may be attributed to not only its antiplatelet effect, but also pleiotropic effects. The pleiotropic effects include inhibiting smooth muscle proliferation and inflammation, inhibiting superoxide formation and oxidative cell death, vasodilating effect by increased production of nitric oxide, and increasing cerebral blood flow and reducing infarct volume as reported from an animal model. ${ }^{21-27}$ These pleiotropic effects with cilostazol are thought to contribute to its prevention of secondary vascular events. Our study also demonstrated that cilostazol was still efficacious and safe for secondary prevention of stroke in Asian patients with PAD.

The effectiveness of aspirin or clopidogrel for prevention of secondary vascular events has been well established in highrisk patients. ${ }^{11}$ In the CAPRIE trial, clopidogrel was significantly superior to aspirin, with $8.7 \%$ risk reduction of major ischemic vascular events and death in all study patients and $23.8 \%$ risk reduction in the subgroup of PAD patients. ${ }^{12} \mathrm{Sim}-$ ilarly, our results showed that clopidogrel significantly reduced by $27 \%$ the risk of all recurrent stroke with more safety than aspirin for Taiwanese patients with PAD. However, the combination of aspirin and clopidogrel was not more effective in preventing major vascular events but rather had more risk of life-threatening bleeding than aspirin alone in the CHARISMA trial ${ }^{28}$ or clopidogrel alone in the MATCH trial. ${ }^{29}$ In the post hoc analysis of the patients with symptomatic or asymptomatic PAD in the CHARISMA trial, clopidogrel plus aspirin provided some benefit over aspirin alone in reducing the rate of $\mathrm{MI}(2.3 \%$ vs. $3.7 \%, \mathrm{P}=0.028)$ and hospitalization for ischemic events $(16.5 \%$ vs. $20.1 \%, \mathrm{P}=0.011){ }^{30}$ There are no large clinical trials investigating the efficacy of secondary prevention of major vascular events by comparing cilostazolbased combination therapy with aspirin or clopidogrel alone. One small pilot study with 76 patients, comparing cilostazol plus aspirin with aspirin alone, showed that the combination therapy had less neurological deterioration within 14 days in patient with acute ischemic stroke in Japan. ${ }^{31}$ In our study, cilostazol-based combination therapy seemed not to provide additional benefit to the prevention of recurrent stroke, but instead a trend to increased hemorrhagic events, especially hemorrhagic stroke. Because of the small number of cases in the combination group, a future large clinical trial may be needed to evaluate the efficacy and safety of cilostazol-based combination treatment in patients with PAD and CVD.

Vitamin K antagonists, such as warfarin, have for more than 50 years been the most effective antithrombotic therapy for the prevention of ischemic stroke in patients with AF. ${ }^{32}$ Recently, several novel oral anticoagulants, such as direct thrombin or factor Xa inhibitors, demonstrated efficacy and safety and were well tolerated in patients with nonvalvular AF in Asian populations. ${ }^{33-36}$ In our study, patients with AF were significantly in the warfarin group than in the other treatment groups. We suspect warfarin might be prescribed for the prevention of cardiac embolization in patients with $P A D$ and $A F$. The European Society of Cardiology guidelines recommend the use of the new ' $\mathrm{CHA}_{2} \mathrm{DS}_{2}-\mathrm{VASc}$ ' score to evaluate the risk for stroke in patients with $\mathrm{AF} .{ }^{37}$ In Taiwan, a nationwide cohort study showed the odds ratio of ischemic stroke was 1.814 for patient with PAD and nonvalvular AF. ${ }^{38}$ Even though the mean level of the international normalized ratio was 1.9 in Chinese patients under long-term warfarin treatment, the incidence of bleeding events remains of concern. ${ }^{39}$ In our study, warfarin did not reduce the risk of recurrent stroke and AMI, and there seemed to be more hemorrhagic events in CVD patients with PAD in that treatment group. 


\section{Study Limitations}

First, the NHI provide the population base database which may have possible disease misclassification and influence diagnostic codes for increasing payment to hospitals and affect the medication of administration. For example, if patients had ether specific or non-specific symptoms, physicians might arrange some image or laboratory examination and wrote some disease codes. Maybe, some patients did not have any "really" diseases. Thus, simply diagnostic codes did not confirm what diseases patients did have. It was a reliable method that we identified PAD with CVD subjects by not only the diagnostic code but also related medication. ${ }^{40}$ Second, we did not know the real data for ABI, carotid intima-media thickness, NIH Stroke Scale (NIHSS), and modified Rankin Scale (mRS). ${ }^{8-10}$ These factors may affect the severity of stroke and major vascular outcomes. Third, because of the lack of echocardiographic assessment, we could hardly distinguish cardioembolic from non-cardioembolic stroke. Fourth, despite the populationbased database, this study did not have any randomization, which may have introduced some selection bias in this study. The safety and efficacy of cilostazol in ischemic stroke patients with peripheral arterial disease study (SPAD, Clinical trial.gov, NCT01188824), a randomized, double-blind, ongoing trial in Taiwan, may provide additional information of cilostazol's benefit on the stroke prevention.

\section{Conclusions}

Similar to previous landmark trials such as CSPS, CSPS 2, CASISP and CAPRIE, we found that cilostazol and clopidogrel are more efficacious and safer than aspirin for the secondary prevention of stroke in CVD patients with PAD. However, warfarin provided no additional benefit than aspirin alone in our study population. Further randomized controlled trials are necessary to confirm our findings.

\section{Acknowledgments}

This study is based in part on data from the National Health Insurance Research Database provided by the Bureau of National Health Insurance, Department of Health and managed by National Health Research Institutes (Registered no. 98178). The interpretation and conclusions contained herein do not represent those of the Bureau of National Health Insurance, Department of Health or National Health Research Institutes. The authors also thank the Statistical Analysis Laboratory, Department of Internal Medicine, Kaohsiung Medical University Hospital for their assistance.

\section{References}

1. Directorate-General of Budget, Accounting and Statistics Executive Yuan, Republic of China. Health. In: Statistical Yearbook of the Republic of China. 2010; 114.

2. Jeng JS, Su TC. Epidemiological studies of cerebrovascular diseases and carotid atherosclerosis in Taiwan. Acta Neurol Taiwan 2007; 16: $190-202$.

3. Chang KC, Tseng MC. Costs of acute care of first-ever ischemic stroke in Taiwan. Stroke 2003; 34: e219-e221.

4. Hu HH, Sheng WY, Chu FL, Lan CF, Chiang BN. Incidence of stroke in Taiwan. Stroke 1992; 23: 1237-1241.

5. Yip PK, Jeng JS, Lee TK, Chang YC, Huang ZS, Ng SK, et al. Subtypes of ischemic stroke: A hospital-based stroke registry in Taiwan (SCAN-IV). Stroke 1997; 28: 2507-2512.

6. Uchiyama S, Goto S, Matsumoto M, Nagai R, Origasa H, Yamazaki $\mathrm{T}$, et al. Cardiovascular event rates in patients with cerebrovascular disease and atherothrombosis at other vascular locations: Results from 1 -year outcomes in the Japanese reach registry. J Neurol Sci 2009; 287: $45-51$.

7. Bhatt DL, Steg PG, Ohman EM, Hirsch AT, Ikeda Y, Mas JL, et al. International prevalence, recognition, and treatment of cardiovascular risk factors in outpatients with atherothrombosis. JAMA 2006; 295: $180-189$.

8. Li C, Wang H, Chen S, Chen Y, Chiang Y, Hsu H. High risk for future events in acute stroke patients with an ankle-brachial index less than 0.9. Acta Cardiol Sin 2012; 28: 17-24.

9. Nakano T, Ohkuma H, Suzuki S. Measurement of ankle brachial index for assessment of atherosclerosis in patients with stroke. Cerebrovasc Dis 2004; 17: 212-217.

10. Meves SH, Diehm C, Berger K, Pittrow D, Trampisch HJ, Burghaus I, et al. Peripheral arterial disease as an independent predictor for excess stroke morbidity and mortality in primary-care patients: 5-year results of the getABI study. Cerebrovasc Dis 2010; 29: 546-554.

11. Antithrombotic Trialists' Collaboration. Collaborative meta-analysis of randomised trials of antiplatelet therapy for prevention of death, myocardial infarction, and stroke in high risk patients. BMJ 2002; 324: $71-86$.

12. CAPRIE Steering Committee. A randomised, blinded, trial of clopidogrel versus aspirin in patients at risk of ischaemic events (CAPRIE): CAPRIE steering committee. Lancet 1996; 348: 1329-1339.

13. Umekawa H, Tanaka T, Kimura Y, Hidaka H. Purification of cyclic adenosine monophosphate phosphodiesterase from human platelets using new-inhibitor sepharose chromatography. Biochem Pharmacol 1984; 33: 3339-3344.

14. Hirsch AT, Haskal ZJ, Hertzer NR, Bakal CW, Creager MA, Halperin JL, et al. ACC/AHA 2005 Practice Guidelines for the Management of Patients With Peripheral Arterial Disease (Lower Extremity, Renal, Mesenteric, and Abdominal Aortic): A Collaborative Report From the American Association for Vascular Surgery/Society for Vascular Surgery, Society for Cardiovascular Angiography and Interventions, Society for Vascular Medicine and Biology, Society of Interventional Radiology, and the ACC/AHA Task Force on Practice Guidelines (Writing Committee to Develop Guidelines for the Management of Patients With Peripheral Arterial Disease): Endorsed by the American Association of Cardiovascular and Pulmonary Rehabilitation; National Heart, Lung, and Blood Institute; Society for Vascular Nursing; Transatlantic Inter-society Consensus; and Vascular Disease Foundation. Circulation 2006; 113: e463-e654.

15. Shinohara Y, Katayama Y, Uchiyama S, Yamaguchi T, Handa S, Matsuoka K, et al. Cilostazol for prevention of secondary stroke (CSPS 2): An aspirin-controlled, double-blind, randomised non-inferiority trial. Lancet Neurol 2010; 9: 959-968.

16. Gotoh F, Tohgi H, Hirai S, Terashi A, Fukuuchi Y, Otomo E, et al. Cilostazol stroke prevention study: A placebo-controlled doubleblind trial for secondary prevention of cerebral infarction. J Stroke Cerebrovasc Dis 2000; 9: 147-157.

17. Cosmi B, Conti E, Coccheri S. Anticoagulants (heparin, low molecular weight heparin and oral anticoagulants) for intermittent claudication. Cochrane Database Systematic Rev 2001; (3): CD001999.

18. Lin LJ, Cheng MH, Lee CH, Wung DC, Cheng CL, Kao Yang YH. Compliance with antithrombotic prescribing guidelines for patients with atrial fibrillation--a nationwide descriptive study in Taiwan. Clin Ther 2008; 30: 1726-1736.

19. Huang Y, Cheng Y, Wu J, Li Y, Xu E, Hong Z, et al. Cilostazol as an alternative to aspirin after ischaemic stroke: A randomised, double-blind, pilot study. Lancet Neurol 2008; 7: 494-499.

20. Lee YS, Bae HJ, Kang DW, Lee SH, Yu K, Park JM, et al. Cilostazol in acute ischemic stroke treatment (CAIST trial): A randomized double-blind non-inferiority trial. Cerebrovasc Dis 2011; 32: 65-71.

21. Takahashi S, Oida K, Fujiwara R, Maeda H, Hayashi S, Takai H, et al. Effect of cilostazol, a cyclic AMP phosphodiesterase inhibitor, on the proliferation of rat aortic smooth muscle cells in culture. J Cardiovasc Pharmacol 1992; 20: 900-906.

22. Otsuki M, Saito H, Xu X, Sumitani S, Kouhara H, Kurabayashi M, et al. Cilostazol represses vascular cell adhesion molecule-1 gene transcription via inhibiting NF-kappaB binding to its recognition sequence. Atherosclerosis 2001; 158: 121-128.

23. Kwon SU, Cho YJ, Koo JS, Bae HJ, Lee YS, Hong KS, et al. Cilostazol prevents the progression of the symptomatic intracranial arterial stenosis: The multicenter double-blind placebo-controlled trial of cilostazol in symptomatic intracranial arterial stenosis. Stroke 2005; 36: $782-786$.

24. Shin HK, Kim YK, Kim KY, Lee JH, Hong KW. Remnant lipoprotein particles induce apoptosis in endothelial cells by NAD(P)H oxidase-mediated production of superoxide and cytokines via lectinlike oxidized low-density lipoprotein receptor-1 activation: Prevention by cilostazol. Circulation 2004; 109: 1022-1028.

25. Park SY, Lee JH, Kim CD, Lee WS, Park WS, Han J, et al. Cilostazol suppresses superoxide production and expression of adhesion molecules in human endothelial cells via mediation of cAMP-dependent protein kinase-mediated maxi-K channel activation. J Pharmacol Exp Ther 2006; 317: $1238-1245$.

26. Hashimoto A, Miyakoda G, Hirose Y, Mori T. Activation of endothelial nitric oxide synthase by cilostazol via a cAMP/protein kinase 
A- and phosphatidylinositol 3-kinase/Akt-dependent mechanism. Atherosclerosis 2006; 189: 350-357.

27. Yuzawa I, Yamada M, Fujii K. An oral administration of cilostazol before focal ischemia reduces the infarct volume with delayed cerebral blood flow increase in rats. J Stroke Cerebrovasc Dis 2008; 17: $281-286$

28. Bhatt DL, Fox KA, Hacke W, Berger PB, Black HR, Boden WE, et al. Clopidogrel and aspirin versus aspirin alone for the prevention of atherothrombotic events. N Engl J Med 2006; 354: 1706-1717.

29. Diener HC, Bogousslavsky J, Brass LM, Cimminiello C, Csiba L, Kaste M, et al. Aspirin and clopidogrel compared with clopidogrel alone after recent ischaemic stroke or transient ischaemic attack in high-risk patients (MATCH): Randomised, double-blind, placebocontrolled trial. Lancet 2004; 364: 331-337.

30. Cacoub PP, Bhatt DL, Steg PG, Topol EJ, Creager MA. Patients with peripheral arterial disease in the CHARISMA trial. Eur Heart J 2009; 30: $192-201$.

31. Nakamura T, Tsuruta S, Uchiyama S. Cilostazol combined with aspirin prevents early neurological deterioration in patients with acute ischemic stroke: A pilot study. J Neurol Sci 2012; 313: 22-26.

32. Fuster V, Ryden LE, Cannom DS, Crijns HJ, Curtis AB, Ellenbogen KA, et al. ACC/AHA/ESC 2006 Guidelines for the Management of Patients with Atrial Fibrillation: A report of the American College of Cardiology/American Heart Association Task Force on Practice Guidelines and the European Society of Cardiology Committee for Practice Guidelines (Writing Committee to Revise the 2001 Guidelines for the Management of Patients With Atrial Fibrillation): Developed in collaboration with the European Heart Rhythm Association and the Heart Rhythm Society. Circulation 2006; 114: e257e354.
33. Ogawa S, Shinohara Y, Kanmuri K. Safety and efficacy of the oral direct factor $x a$ inhibitor apixaban in Japanese patients with nonvalvular atrial fibrillation: the ARISTOTLE-J study. Circ J 2011; 75: $1852-1859$.

34. Ogawa S, Koretsune Y, Yasaka M, Aizawa Y, Atarashi H, Inoue H, et al. Antithrombotic therapy in atrial fibrillation: Evaluation and positioning of new oral anticoagulant agents. Circ J 2011; 75: 15391547.

35. Hori M, Matsumoto M, Tanahashi N, Momomura SI, Uchiyama S, Goto S, et al. Rivaroxaban vs. warfarin in Japanese patients with atrial fibrillation. Circ J 2012; 76: 2104-2111.

36. Yamashita T, Koretsune Y, Yasaka M, Inoue H, Kawai Y, Yamaguchi $\mathrm{T}$, et al. Randomized, multicenter, warfarin-controlled phase II study of edoxaban in Japanese patients with non-valvular atrial fibrillation. Circ J 2012; 76: 1840-1847.

37. Camm AJ, Kirchhof P, Lip GY, Schotten U, Savelieva I, Ernst S, et al Guidelines for the management of atrial fibrillation: The Task Force for the Management of Atrial Fibrillation of the European Society of Cardiology (ESC). Europace 2010; 12: 1360-1420.

38. Lin LY, Lee CH, Yu CC, Tsai CT, Lai LP, Hwang JJ, et al. Risk factors and incidence of ischemic stroke in Taiwanese with nonvalvular atrial fibrillation: A nation wide database analysis. Atherosclerosis 2011; 217: 292-295.

39. Chenhsu RY, Chiang SC, Chou MH, Lin MF. Long-term treatment with warfarin in Chinese population. Ann Pharmacother 2000; 34: $1395-1401$.

40. Assaf AR, Lapane KL, McKenney JL, Carleton RA. Possible influence of the prospective payment system on the assignment of discharge diagnoses for coronary heart disease. N Engl J Med 1993; 329: $931-935$. 